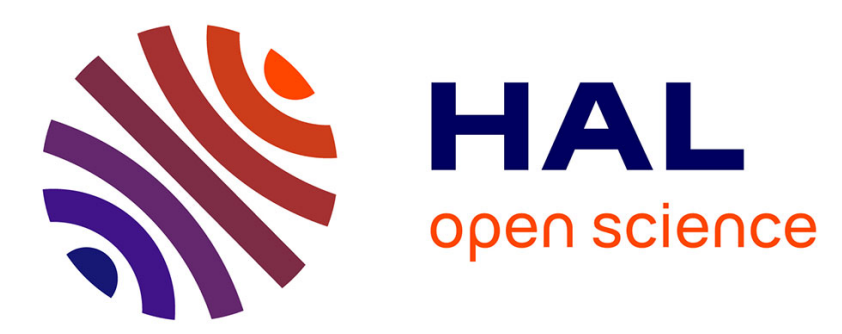

\title{
Compressive-Sensing-based Multidimensional Doppler signal analysis for fetal activity monitoring
}

\author{
Adrian Basarab, Céline Quinsac, Jean-Marc Girault, Denis Kouamé
}

\section{To cite this version:}

Adrian Basarab, Céline Quinsac, Jean-Marc Girault, Denis Kouamé. Compressive-Sensing-based Multidimensional Doppler signal analysis for fetal activity monitoring. IEEE International Ultrasonics Symposium - US 2014, Sep 2014, Chicago, United States. pp. 1073-1076. hal-01147233

\section{HAL Id: hal-01147233 \\ https://hal.science/hal-01147233}

Submitted on 30 Apr 2015

HAL is a multi-disciplinary open access archive for the deposit and dissemination of scientific research documents, whether they are published or not. The documents may come from teaching and research institutions in France or abroad, or from public or private research centers.
L'archive ouverte pluridisciplinaire HAL, est destinée au dépôt et à la diffusion de documents scientifiques de niveau recherche, publiés ou non, émanant des établissements d'enseignement et de recherche français ou étrangers, des laboratoires publics ou privés. 


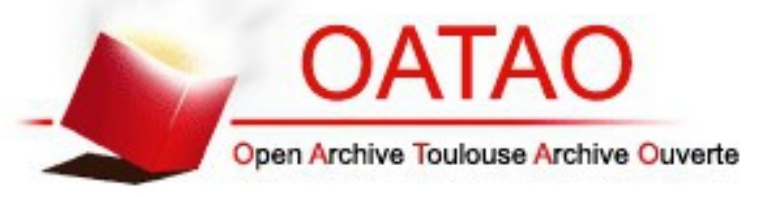

\section{Open Archive TOULOUSE Archive Ouverte (OATAO)}

OATAO is an open access repository that collects the work of Toulouse researchers and makes it freely available over the web where possible.

This is an author-deposited version published in : http://oatao.univ-toulouse.fr/ Eprints ID : 13081

To link to this article : DOI :10.1109/ULTSYM.2014.0263

URL : http://dx.doi.org/10.1109/ULTSYM.2014.0263

To cite this version : Basarab, Adrian and Quinsac, Céline and Girault, Jean-Marc and Kouamé, Denis Compressive-Sensing-based Multidimensional Doppler signal analysis for fetal activity monitoring. (2014) In: IEEE International Ultrasonics Symposium - US 2014, 3 September 2014 - 6 September 2014 (Chicago, United States).

Any correspondance concerning this service should be sent to the repository administrator: staff-oatao@,listes-diff.inp-toulouse.fr 


\section{Compressive-Sensing-based multidimensional Doppler signal analysis for fetal activity monitoring}

\author{
Adrian Basarab \\ University of Toulouse \\ IRIT UMR CNRS 5505 \\ Toulouse, France
}

\author{
Céline Quinsac \\ University of Toulouse \\ IRIT UMR CNRS 5505 \\ Toulouse, France
}

\author{
Jean-Marc Girault \\ University of Tours \\ François Rabelais \\ INSERM U930 \\ Tours, France
}

\author{
Denis Kouamé \\ University of Toulouse \\ IRIT UMR CNRS 5505 \\ Toulouse, France
}

\begin{abstract}
Fetal activity monitoring is an important part of monitoring at-risk pregnancies and labor. Fetal activity parameters (FAP) consist of fetal heart rate (FHR), fetal movements (FM) rate, fetal tone, fetal breathing (FB) and movement. FAP monitoring is to date an open challenge for mainly two reasons. First, the estimation of FAP is highly time consuming and thus cannot be used routinely. Second, part of FAP such as FM estimation is sometimes subjective (mothers are asked to count the fetal movements) and inaccurate. For this purpose, we developed a $2 \mathrm{MHz}$ pulsed wave ultrasound Doppler system, consisting of 12 transducers with 5 adjustable gates. The Doppler signals were sampled at $1 \mathrm{KHz}$. Several recent papers have shown the accuracy of our system. However, its counterpart is the huge number of signals necessary to estimate the FAP. Specifically, each millisecond, 60 Doppler samples are acquired. In order to reduce the volume of the acquired data and to accelerate the FAP estimation rate, we propose herein to investigate the interest of compressive sensing (CS) techniques to our application.
\end{abstract}

\section{INTRODUCTION}

Electronic fetal monitoring may be required during the whole pregnancy to closely monitor certain fetal and maternal disorders such as pre-eclampsia, diabetes, post-term pregnancy (over 42 weeks) and antepartum hemorrhage, fetal growth retardation, prematurity and abnormal artery velocity [1][2].

Many different types of fetal monitoring systems exist nowadays. Ultrasound B-mode scanners are usually used to obtain fetal measurements in order to detect abnormal growth. Doppler measurements are employed to study blood circulation between mother and fetus, as well as in the umbilical cord. The cardiotocogram, continuous or discontinuous electronic fetal heart rate monitoring device, is the reference in the field of Electronic Fetal Monitoring (EFM). However, while it accurately measures the FHR, it is less adapted to inform about fetal movements. All these measurements are performed at the beginning and periodically during the pregnancy.

Ultrasound (US) Doppler provides both detection of the FHR and movements of the fetus, thus giving more information on the fetal well-being. Widely used for FHR monitoring, these mono or bi-transducer systems using continuous or pulsed ultrasound waves provide partial automated detection of movements [3] or fetal breathing [4] [5].

In this context, we have recently developed a more complete system: a $2 \mathrm{MHz}$ pulsed wave ultrasound Doppler system, consisting of 12 transducers with 5 adjustable gates. The resulting Doppler signals were sampled at $1 \mathrm{KHz}$. The system allows to access to the FHR, the movements of the fetus (and related parameters) and also the spontaneous rhythms. These parameters are referred to as fetal activity parameters (FAP). It has been shown that this system is very accurate [6]. However, its counterpart is the huge number of signals necessary to estimate the FAP. Specifically, each millisecond, 60 complex value Doppler samples are acquired. In order to reduced the volume of the data during acquisition and accelerate the FAP estimation rate, we propose to investigate the applicability of compressive sensing (CS) in the context of our Doppler system. Compressed sensing (or compressive sampling) is a mathematical framework that allows the reconstruction of sparse signals or images (or compressible in a known basis) from far fewer measurements than predicted by the classical Nyquist-Shannon theory [7]. In medical ultrasound, several application schemes of the CS theory have been proposed, in US imaging (see [8] for a review of existing methods) or in pulsed Doppler [9][10].

The reminder of this paper is organized as follows. First, we report the most important technical information about our Doppler system. Next, the basics of CS theory and our application scheme to decrease the data acquired with our system are given. Finally, results showing the FAP estimation with fully sampled and with CS reconstructed Doppler signals are reported.

\section{MATERIAL}

\section{A. The system}

A Doppler system has been developed for fetal activity monitoring (Surfoetus system built by Althais Technologies ${ }^{\mathrm{TM}}$ ). This Doppler system consists of twelve $2 \mathrm{MHz}$ transducers (3 groups of 4 transducers) with five gates. This device includes three electronic pulsed Doppler boards, a data acquisition board, and the three groups of transducers.

Body exploration is performed with all the 12 transducers. Five adjustable gates ranging from 2 to 14 centimeters were explored. These gates were independently adjustable in depth.

\section{B. Transducer arrangement}

Transducers were divided into three groups A, B and C. Group A was used to explore the fetal thorax, investigating 
fetal heart rate and breathing movements, group B the upper limbs and group $\mathrm{C}$ the lower limbs. The twelve transducers monitor the activity of all the regions of interest in the fetus. The ultrasound beam of the transducers was as wide as possible to maximize the explored region.

\section{Electronic Doppler control}

The electronic module consisted of three boards, each board driving a group of four transducers. The emission signal was sinusoid, with frequency $f_{0}=2 \mathrm{MHz}$. A multiplexer selected one of the four transducers driven by the board. The received signal was first amplified by a low-noise amplifier, then by a deep compensated amplifier in order to compensate for the strong attenuation of the deepest gates. Finally the signal was band-pass filtered (center frequency $f_{0}$ ). After complex inphase demodulation, the components were sampled sequentially and converted.

All three boards worked simultaneously, a transducer emitting every millisecond. Pulse Repetition Frequency (PRF) was $1 k H z$, and a transducer was activated for $250 \mu s$, see Fig. 2. This time is sufficient to carry out five samplings of the direct and squared components necessary to explore five gates. Sensors of each group work separately, emitting and receiving the ultrasound beam in turn.

\section{Ultrasound Doppler signal parameters}

When an ultrasound wave with frequency $f_{0}$ is emitted from a transducer towards a moving target, the frequency of the received backscattered wave is $f_{0}+f_{d}$. After complex inphase demodulation, the quadrature analytical Doppler signal can be written as:

$$
x_{k}(t)=\beta_{k}(t) \exp j\left[2 \pi f_{d k}(t)+\varphi_{k}(t)\right]=I_{k}(t)+j Q_{k}(t)
$$

where $k=1, \ldots K$ (in our case $K=60$ ), $\beta_{k}(t)$ is the random magnitude of the Doppler signal, depending on the characteristics of the transducers and the measurement area, and $\varphi(t)$ is the random phase, depending on the positions of the scatterers in the explored medium. Note that $k$ runs from 1 to $K=60$ as with our multi-Doppler system, 12 transducers are used for 5 different depths, thus a total of 60 complex value Doppler signals. The frequency shift $f_{d}$ (Doppler frequency), can be written as:

$$
f_{d k}(t)=\frac{2 f_{0}}{c} v_{k}(t) \cos \theta_{k}
$$

where $v_{k}=\left|\overrightarrow{v_{k}}\right|$ and $\overrightarrow{v_{k}}$ is the target velocity, $\theta_{k}$ is the angle between the ultrasound beam and the vector $\overrightarrow{v_{k}}$, and $c$ is the ultrasound wave velocity in the explored medium. Note that angles $\theta_{k}$ do not have to be evaluated precisely here, since the purpose is to extract variations in fetal heart rate.

Each Doppler signal contains a large amount of information concerning target movement (velocity, position) via the Doppler frequency and the overall phase. This overall phase $\phi_{k}(t)=2 \pi f_{d k}(t)+\varphi_{k}(t)$ directly provides the position or displacement $\Delta z$ of the target [11]:

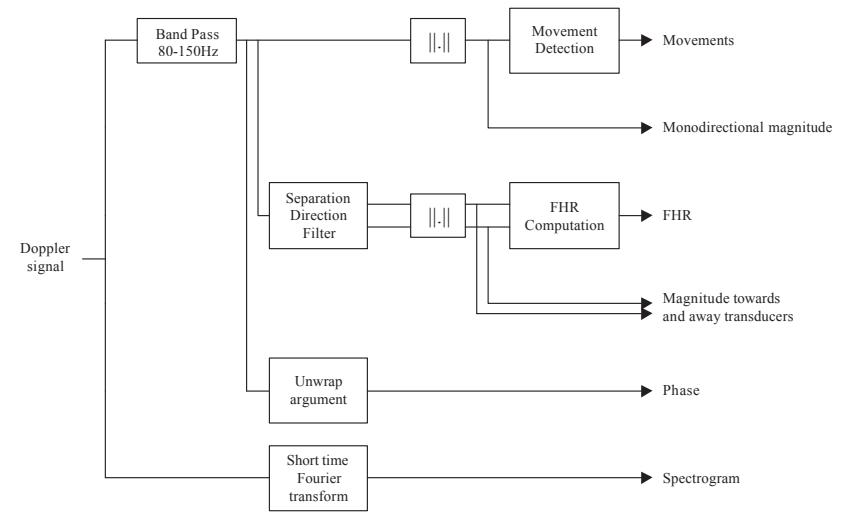

Fig. 1. Pre-processing of Doppler signal prior to Fetal activity parameter estimation.

$$
\phi=\frac{4 \pi f_{0}}{c} \Delta z=\frac{4 \pi}{\lambda} \Delta z
$$

where $\lambda=\frac{c}{f_{0}}$ is the wave length. The velocity of the target is proportional to the Doppler frequency, whose estimation has been largely addressed in the literature, e.g [12].

Finally, the magnitude of the backscattered signal depends on the characteristics of the transducers and the nature of the scatterers. To follow the magnitude of movements towards and away from the transducer separately, a direction separation filter is necessary, consisting of eliminating both positive frequency components and negative frequency components. This separation filter thus gives two (forwards and backwards) signals. As mentioned above, direction information can also be accessed through the overall phase.

The estimation of the different FAP parameters (fetal heart rate (FHR), fetal movements (FM) rate, fetal tone, fetal breathing (FB) movement) are thus based on the Doppler signals. The Doppler signals are preprocessed to access the FAP, as shown in Fig. 1.

From these pre-processing, FAP can be estimated using time and/or frequency techniques, as shown in [13], [14], [15], [16] and references therein.

\section{E. Data storage}

Data was recorded directly on the computer hard drive using a 12 bit data acquisition board, storing data at a speed of 240 $\mathrm{KB}$ per second. Each minute of recording uses thus more than $22 \mathrm{MB}$. The data is recorded during $30 \mathrm{~min}$ and post-processed in order to estimate the FAP. We may remark that despite its accuracy, our system requires the acquisition, storage and processing of a large quantity of data. In order to reduced the volume of the data during the acquisition and accelerate the FAP estimation rate, we propose in this paper to investigate the applicability of compressive sensing (CS) to our acquisition scheme. 


\section{PROPOSED COMPRESSED SENSING SCHEME FOR MULTIDIMENSIONAL DOPPLER}

\section{A. Basics of compressed sensing}

Compressed sensing (or compressive sampling) is a mathematical framework that offers guarantees for "perfect" signal or image reconstruction from far less measurements than the number predicted by Nyquist-Shannon theorem [7]. Many theoretical results on CS may be found in the literature. We give hereafter only the basics, so that the reader may understand the interest and the requirements of CS.

The first important issue in CS is the sparsity of the signal or of the image to reconstruct (or to recover). Specifically, a vector $x$ containing $N$ elements is $S$-sparse in a basis $\psi$ if the vector $\alpha$ such as $x=\psi \alpha$ has only $S<N$ nonzero elements.

The second important aspect of CS is the concept of incoherent measurements. Let us denote by $y$ the vector containing these measurements. It is related to $x$ by $y=R \Phi x$. $\Phi$, an $N$ by $N$ real value matrix called the measurement matrix, generates linear combinations of elements in $x$. $R$ is called the restriction matrix and is of size $M$ by $N$, with $M<<N$. It only contains zeros and ones (one elements equal to 1 in each row). $R$ chooses a subset of $M$ measurements out of the $N$ possible values of $\Phi x$. It should be noted that the success of CS largely depends on the choice of $\Phi$ and $R$. They have to ensure incoherence (see [17] for technical details about the concept of incoherence) between the sampling vectors and the vectors forming the sparsity basis $\psi$. A typical choice providing maximal incoherence, not always easy to use in practice, is a matrix $R \Phi$ formed by $M$ random (e.g. Gaussian) vectors.

Given the measurement model $y=R \Phi \psi \alpha$ (an underdetermined system of $M$ equations with $N$ unknowns), one may recover $\alpha$ (and consequently $x$ ) by solving the following $\ell_{1}$ minimization problem:

$$
\min \|\widehat{\alpha}\|_{1} \quad \text { subject to } y=R \Phi \psi \widehat{\alpha}
$$

\section{B. Compressed sensing for fetal activity analysis}

As explained in the section describing our multi Doppler system, the FAP are estimated using $K=60$ Doppler signals called $x_{k}$ in equation (1), corresponding to the 12 transducers and to the 5 explored depths. The transducers are grouped by 4 , in three groups working simultaneously and in the same manner. We show in Fig. 2 the timing corresponding to the acquisition of 20 Doppler signals of one of the three groups of transducers. Each transducer acquires 5 samples during a time interval of $250 \mu \mathrm{s}$, each one corresponding to one depth. For instance, the first transducer delivers during the first $250 \mu \mathrm{s}$ when it is active the samples $\left[x_{1}(1) x_{2}(1) x_{3}(1) x_{4}(1) x_{5}(1)\right]$. After $1 \mathrm{~ms}$ and during its next activity time interval of $250 \mu \mathrm{s}$, it will acquire the samples $\left[x_{1}(2) x_{2}(2) x_{3}(2) x_{4}(2) x_{5}(2)\right]$.

Given the sampling rate and the duration of the acquisition, an important amount of data is generated. The idea in this paper is to use the CS framework in order to decrease the number of samples of each Doppler signal. We propose

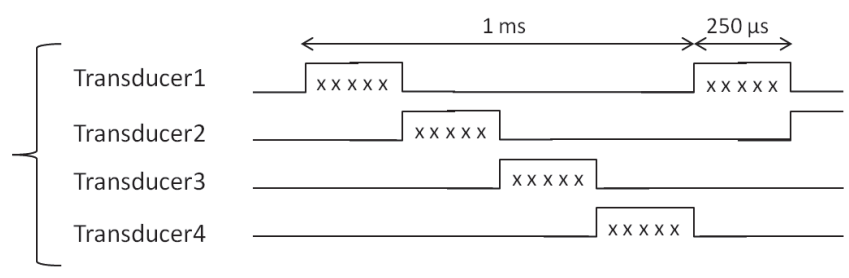

Fig. 2. Timing showing the classical way of acquiring the Doppler signals with our system. In each time laps of $250 \mu \mathrm{s}$ when a transducer is active, it acquires 5 samples of 5 Doppler signals, highlighted by the $x$ markers.

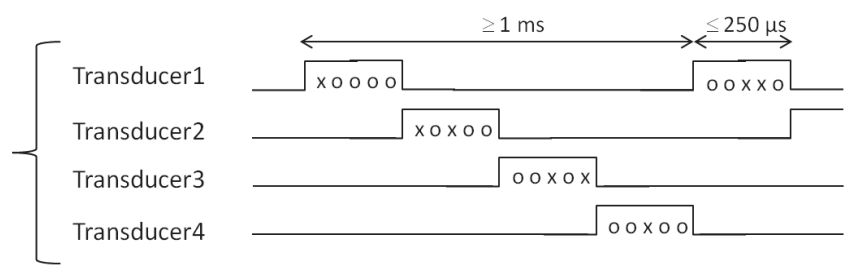

Fig. 3. Timing showing the proposed CS way of acquiring the Doppler signals with our system. In each time laps when a transducer is active, it acquires less than 5 samples, highlighted by the $x$ markers. The $o$ markers indicate that that sample is not acquired at all.

herein to use an identity matrix as measurement matrix $\Phi$ for all the $K=60$ Doppler signals. With this choice of a structured measurement matrix, it is shown in [18] that the restriction matrix $R$ has to be designed such as $M$ elements are randomly chosen from signals $x_{k}$. Our measurement model, as highlighted in Fig. 3, is as follows:

$$
y_{k}=R_{k} x_{k}
$$

for $k$ running from 1 to $K=60$. In (5), the restriction matrices $R_{k}$ are of size $M$ by $N$ and have all the elements equal to 0 , except one element by row equal to 1 . The positions of the ones are uniformly random distributed. As shown in Fig. 3 , this way of skipping some of the Doppler signal samples, potentially decreases the length of the activation period of each sensor, initially of $250 \mu \mathrm{s}$. Consequently, the sampling frequency whose initial value is $1 \mathrm{kHz}$ may be decreased, thus generating less data per Doppler signal.

We propose in this paper to recover jointly all the $K$ Doppler signals $x_{k}$ from the measurements $y_{k}$. Let us denote by $X$ the matrix formed by juxtaposing the the $K$ Doppler signals $x_{k}$. As we have explained previously, one of the key points in the CS framework is the sparsity assumption of the signals to recover. Herein, we consider the 2D Fourier transform $(\mathcal{F})$ of $X$ to be sparse [19]. The recovery of the Doppler signals follows the optimization process in (6). It should be noted that the value of $\lambda$, the parameter weighting for sparsity, has been automatically tuned using a reweighted $\ell_{1}$ approach [20]. 

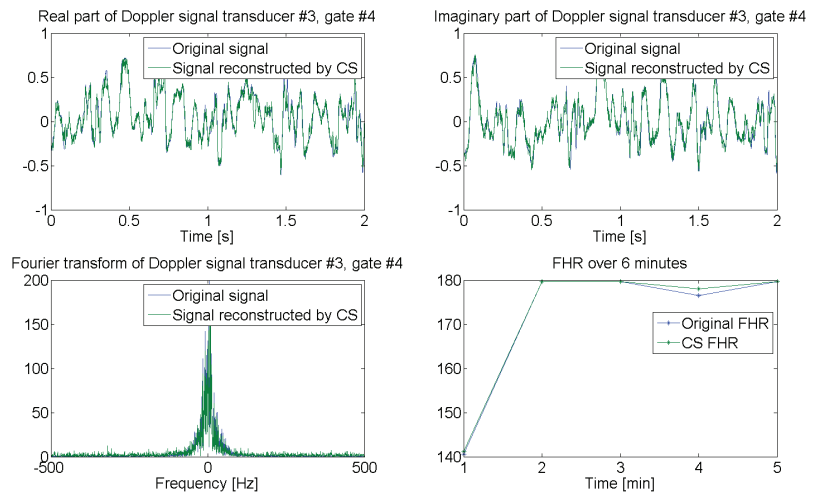

Fig. 4. Reconstruction result of a Doppler signal from $30 \%$ of the original sample (real and imaginary part, Fourier transform) and FHR estimated with the original and reconstructed signals.

$$
\min \left(\|\mathcal{F} X\|_{1}+\lambda \sum_{k=1}^{K}\left\|y_{k}-R_{k} x_{k}\right\|_{2}^{2}\right)
$$

\section{RESUltS}

The results of our method allowed the reconstruction of US Doppler signals from $33 \%$ of the initial time samples. That is, $M=N / 3$. One reconstruction result is reported in Fig. 4. The result shows the accuracy of the reconstruction, corresponding to a root mean square error of less than $10 \%$. We may also remark that the FHR estimated with the original and CSreconstructed Doppler signals are very close, corresponding to a mean square error of 0.58 over 6 minutes of acquisition. Note that this is true for all the FAP usually calculated with our system.

\section{CONCLUSION}

We have shown in this paper the applicability of CS framework to a multidimensional Doppler system. Used for fetal activity monitoring during pregnancy, this system uses 12 US transducers for 5 different depths, which corresponds to 60 complex value Doppler signals. Given the large amount of data generated, we have proposed a CS scheme aiming to reduce the number of acquired samples and to accelerate the acquisition process. We show that a reduction of $66 \%$ of the initial number of samples does not alter the estimation of the fetal activity parameters such as the fetal heart rate.

\section{ACKNOWLEDGMENT}

The authors would like to thank Althais Technologies and University Hospital of Tours, France.

\section{REFERENCES}

[1] FA Manning, LD Platt, and Sipos L., "Antepartum fetal evaluation: Development of a fetal biophysical profile," Am J Obstet Genicol, vol. 136, pp. 787-795, 1980.

[2] DK James et al., Royal College of Obstetricians and Gynaecologists, The Use of Electronic Fetal Monitoring, The use and interpretation of Cardiotocography in intrapartum fetal surveillance, RCOG Press, 2001.
[3] B. Karlsonn, M. Berson, T.Helgason, R.T. Geirsson, and L. Pourcelot, "Effect of fetal and maternal breathing on the ultrasonic Doppler signal due to fetal heart movement," European journal of ultrasound, vol. 11, pp. 47-52, 2000.

[4] B. Karlsonn, K. Foulquière, K. Kaluzynski, F. Tranquart, A. Fignon, D. Pourcelot, L. Pourcelot, and M. Berson, "The dopfet system : a new ultrasonic Doppler system for monitoring and characterisation of fetal movement," Ultrasound in Med \& Biol., vol. 26, no. 7, pp. 1117-1124, 2000.

[5] Y. Yamakoshi, T. Shimizu, N. Shinozuka, and H. Masuda, "Automated fetal breathing movement detection from internal small displacement measurement," Biomed. Technik, vol. 41, pp. 242-247, 1996.

[6] Sophie Ribes, Denis Kouamé, Iulian Voicu, Morgane FournierMassignan, Franck Perrotin, and François Tranquart, "Support vector machines based multidimensional signals classification for fetal activity characterization," in SPIE Medical Imaging, Feb. 2011, vol. 7968.

[7] E. J. Candes, J. Romberg, and T. Tao, "Robust uncertainty principles: exact signal reconstruction from highly incomplete frequency information," IEEE Transactions on Information Theory, vol. 52, no. 2, pp. 489-509, January 2006.

[8] H. Liebgott, A. Basarab, D. Kouamé, O. Bernard, and D. Friboulet, "Compressive sensing in medical ultrasound," in IEEE International Ultrasonics Symposium (IUS), Dresden (Germany), 2012, pp. 1-6.

[9] S.M.S. Zobly and Y.M. Kakah, "Compressed sensing: Doppler ultrasound signal recovery by using non-uniform sampling amp; random sampling," in Radio Science Conference (NRSC), 2011 28th National, April 2011, pp. 1-9.

[10] J. Richy, D. Friboulet, A. Bernard, O. Bernard, and H. Liebgott, "Blood velocity estimation using compressive sensing," IEEE Transactions on Medical Imaging, vol. 32, no. 11, pp. 1979-1988, 2013.

[11] Jensen J.A., Estimation of blood velocities using ultrasound. A signal processing approach, Cambridge : University Press,, 1996.

[12] D. Kouamé, J-M. Girault, and F. Patat, "High resolution processing techniques for ultrasound Doppler velocimetry in presence of colored noise part 1 : Nonstationary methods," IEEE Trans. Ultrason. Ferro. Frequency. Contr, vol. 50, no. 3, pp. 257-266, 2003.

[13] M. David, M. Hirsch, J. Karin, E. Toledo, and S. Akselrod, "An estimate of fetal autonomic state by time-frequency analysis of fetal heart rate variability," Journal of Applied Physiol,, vol. 102, pp. 1057Û́1064, 2007.

[14] Iulian Voicu, Sébastien Ménigot, Denis Kouamé, and Jean-Marc Girault, "New estimators and guidelines for better use of fetal heart rate estimators with doppler ultrasound devices," Computational and Mathematical Methods in Medicine, vol. 2014.

[15] I. Voicu, JM. Girault, M. Fournier-Massignan, and D. Kouamé, "Robust estimation of fetal heart rate form us doppler signals," in Proceedings of the International Congress on Ultrasonics (ICU 2009), Physics Procedia 3(1):691-699, 2009, vol. 3, pp. 691-699.

[16] D. Rouvre D. Kouamé F. Tranquart L. Pourcelot, "Empirical mode decomposition (emd) for multi-gate, multi-transducer ultrasound doppler fetal heart monitoring," IEEE International Symposium on Signal Processing and Information Technology, vol. 1, Dec. 2005.

[17] E.J. Candes and M.B. Wakin, "An introduction to compressive sampling," Signal Processing Magazine, IEEE, vol. 25, no. 2, pp. 21-30, March 2008.

[18] M.F. Duarte and Y.C. Eldar, "Structured compressed sensing: From theory to applications," Signal Processing, IEEE Transactions on, vol. 59, no. 9, pp. 4053-4085, Sept 2011.

[19] C. Quinsac, A. Basarab, and D. Kouame, "Frequency domain compressive sampling for ultrasound imaging," Advances in Acoustics and Vibration, Advances in Acoustic Sensing, Imaging, and Signal Processing, vol. 12, pp. 1-16, 2012.

[20] EmmanuelJ. Candès, MichaelB. Wakin, and StephenP. Boyd, "Enhancing sparsity by reweighted 11 minimization," Journal of Fourier Analysis and Applications, vol. 14, no. 5-6, pp. 877-905, 2008. 